\title{
The orbital period study and photometric analysis of XY Ursae Majoris
}

\author{
A. Erdem and N. Güdür \\ Department of Physics, Faculty of Arts and Sciences, Canakkale Onsekiz, Mart University, 17100 Canakkale, Turkey \\ e-mail: comummfd@turnet.net.tr or aerdem@bornova.ege.edu.tr
}

Received December 9, 1996; accepted May 9, 1997

\begin{abstract}
Photoelectric observations of the eclipsing binary XY UMa have been carried out in $B$ and $V$ colours at the Ege University Observatory. Combining these newly obtained data with the previous ones available in the literature, the problem pertaining to the orbital period of the system has been investigated through a detailed description of the $\mathrm{O}-\mathrm{C}$ diagram. The sinusoidal and secular changes have been found. Such variations of the orbital period have been examined in terms of three plausible mechanisms, namely (i) a light-time effect due to a third body, (ii) the orbital period modulation due to magnetic activity cycle of a component star, and (iii) a mass loss case in which the ejected material escapes out from the binary system. A combination of a magnetic activity cycle mechanism and a mass loss case is found to reproduce satisfactorily the period changes. Further, the observed photoelectric light curves of the system have been analyzed with the Wilson-Devinney approach. This analysis shows that the asymmetries of the light curves of XY UMa can be interpreted in terms of cool star-spot covering $7 \%$ of the stellar photosphere of the primary component of this binary. The system is found to be a $\beta$ Lyrae type detached binary. The absolute elements of the system are also deduced.
\end{abstract}

Key words: stars: binaries - close - stars: individual — XY UMa - fundamental parameters starspots

\section{Introduction}

$\mathrm{XY} \operatorname{UMa}\left(=\mathrm{HD} 27143=\mathrm{BD}+55^{\circ} 1317=\mathrm{BV} 31\right)$ was firstly noted by Geyer et al. (1955) as an eclipsing binary with a period of $\mathrm{P}=0.4799$. After which Geyer began a prolonged series of photometric observations, the results of which he reported in 1976, 1977, and 1980. He found

Send offprint requests to: A. Erdem that the revolution period of 0.478995 was constant during 20 years (15250 epochs!), and that the average brightness of the binary system changed between 1955 and 1975 in a sinusoidal manner by $0.18 \mathrm{mag}$ in $V$ and $0.20 \mathrm{mag}$ in $B$, indicating a periodic variation of about 28 to 30 yrs. He gave a general explanation of the peculiarities of the photometric light curves of the system following as: the primary component shows a larger star-spot activity, the cycle of which is about 15 yrs (or double that value). So, he held responsible this star-spot activity for the observed asymmetrical light curves. Furthermore, carrying out spectroscopic observations of the system, he gave that the spectral classes of the components of XY UMa as G2$\mathrm{G} 5 \mathrm{~V}+\mathrm{K} 5 \mathrm{~V}$. He also stated that both component are well below their Roche limit configuration. And then, the star was classified as a cool, "short period RS CVn" star by Baliunas \& Vaughan (1985). Indeed, XY UMa, in terms of its chromospheric surface flux, may well be the most active of such systems (Gurzadyan 1987).

During the last decade XY UMa attracted the interest of many observers: the system has been observed intensively in optical (e.g. Geyer 1980; Zeilik et al. 1982; Jassur 1986; Heckert \& Zeilik 1988; Qisheng et al. 1989; Zeilik et al. 1988; Hilditch \& Bell 1994; Jeffries et al. 1995), radio (Drake et al. 1986; Morris \& Mutel 1988), infrared (Arevalo \& Lazaro 1990; Mitrou et al. 1996), ultraviolet (Geyer \& Hoffmann 1981) and X-ray (Bedford \& Geyer 1986; Bedford et al. 1990; Dempsey et al. 1993) domains. Some polarimetric (Geyer \& Metz 1977), spectroscopic (Budding \& Kadouri 1982; Huisong \& Xuefu 1987; Rainger et al. 1991) investigations have also been performed. Gurzadyan \& Cholakyan (1995) investigated the magnesium (MgII) luminosities of XY UMa and other RS CVn stars which have been observed by Dempsey et al. (1993), and concluded that the main contribution in the magnesium emission in RS CVn type systems has purely intercomponent origin.

At least five O-C diagrams have been published upto-date (Geyer 1977; Lorenzi \& Scaltriti 1977; Hall \& Kreiner 1980; Lee 1985; Pojmanski \& Geyer 1990). Each 
Table 1. Data for the variable, comparison, and check stars

\begin{tabular}{|c|c|c|c|c|c|c|c|}
\hline Star & BD No & HD No & $\alpha(\mathbf{1 9 0 0 )}$ & $\delta(\mathbf{1 9 0 0 )}$ & $m_{\mathrm{pg}}$ & $m_{\mathrm{v}}$ & Sp. \\
\hline XY UMa & $+55^{\circ} 1317$ & 27143 & $09^{\mathrm{hr}} 02^{\min } .7$ & $+54^{\circ} 54^{\prime} .9$ & $10^{\mathrm{m}} 4$ & 9.1 & G2-G5 \\
\hline Comparison & $+54^{\circ} 1278$ & 237784 & $09^{\mathrm{hr}} 04^{\mathrm{min}} .2$ & $+54^{\circ} 23^{\prime} .7$ & $10 .{ }^{\mathrm{m}} 0$ & 9.0 & F5 \\
\hline Check & $+54^{\circ} 1275$ & 233599 & $09^{\mathrm{hr}} 07^{\mathrm{min}} .4$ & $+54^{\circ} 0^{\prime} .92$ & 10.7 & 9.0 & $\mathrm{~K} 2$ \\
\hline
\end{tabular}

one shows a large scatter up to a value of 0d02. Geyer (1977) noted that the period of the system seemed to be constant. However some quadratic terms in ephemeris were introduced by Hall \& Kreiner $\left(1980,+2.810^{-11} \cdot E^{2}\right)$ and Qisheng et al. $\left(1989,+1.410^{-11} \cdot E^{2}\right)$. Hall \& Kreiner attributed the long-term variations to the mass-loss from the system. Pojmanski \& Geyer (1990) showed that the $\mathrm{O}-\mathrm{C}$ residuals are strongly correlated with the asymmetry of the light curve (difference between the heights of the maxima was used as its measure) and concluded that the possible explanation for $\mathrm{O}-\mathrm{C}$ variation of the system $\mathrm{XY}$ UMa is the hypothesis of a third-body with $0.28 \mathrm{~m}_{\odot}$ (K5-M2 dwarf) revolving around the close binary. The main difficulty remained unsolved in their work, however, is how could the light-time effect be held responsible from the asymmetry seen in the light curves by ignoring the star-spot activity.

Light curve solutions of the system have been obtained by Geyer (1980), Jassur (1986), Budding \& Zeilik (1987), and Banks \& Budding (1989) from visible photometry, and Arevalo \& Lazaro (1990) from infrared photometry. Many of them analyzed their light curves using the Budding (1977) method and concluded that the large light curve changes of XY UMa is due to stellar star-spot activity of the primary component of this binary.

\section{Observational data}

The system was observed photoelectrically at the Ege University Observatory. The observations were performed in the observational season of 1994. The $48 \mathrm{~cm}$ (f/13) Cassegrain reflector equipped with an unrefrigerated EMI 9781 A photomultiplier tube was used. All observations were made with the $B$ and $V$ filters of the $U B V$ system. A total of 197 observational points were obtained in each colour. $\mathrm{BD}+54^{\circ} 1278$ and $\mathrm{BD}+54^{\circ} 1275$ were used as comparison and check stars, respectively. Data related to the variable, comparison and check stars are given in Table 1. The atmospheric extinction coefficients in each colour for each night were calculated from the observations of the comparison star using conventional methods. Then, all the instrumental differential $B$ and $V$ magnitudes (in the sense variable minus comparison) were corrected for atmospheric extinction. Light and colour curves are shown in Fig. 1 and Fig. 2, respectively. The phases were calculated with the formula (2) which is given in Sect. 3.1.

The shape of the light curves of XY UMa in Fig. 1 are typical of $\beta$ Lyrae type. A pronounced asymmetry is evident in the light curves. This asymmetry is located between the descending and ascending shoulders of the primary minimum. In the literature, the existence of the asymmetry in the light curves of XY UMa has been stated and the responsibility was put on a star-spot activity. The fact that the asymmetry is observed in the phases of secondary minimum and afterwards, and also the unexpected light loss during these phases, might indicate to a star-spot activity on the surface of the primary component. The colour curve in Fig. 2 shows that the system is redder at the primary and slightly bluer at the secondary minimum. So, the spectral type of the secondary component is later than that of the primary.

\section{Orbital period analysis}

\subsection{O-C for minima}

During the observations of XY UMa in this work, times of four primary and two secondary minima were obtained and are given in Table 2 . The $\mathrm{O}-\mathrm{C}_{1}$ values were computed using the following light elements given by Geyer et al. (1955):

Hel Min I JD $=2435216.5011+0.4789944 \cdot E$.

Table 2. Times of minima of XY UMa observed in March 1994

\begin{tabular}{lllcr}
\hline $\begin{array}{l}\text { H JD } \\
\text { 2400000.+ }\end{array}$ & Min & Filter & O-C & O-C 2 \\
\hline 49433.5427 & I & $B$ & 0.0088 & -0.0001 \\
49433.5436 & I & $V$ & 0.0097 & 0.0008 \\
49436.4161 & I & $B$ & 0.0082 & -0.0007 \\
49436.4175 & I & $V$ & 0.0096 & 0.0007 \\
49443.393 & II & $V$ & 0.0397 & $\cdots$ \\
49443.395 & II & $B$ & 0.0417 & $\cdots$ \\
\hline
\end{tabular}

The $\mathrm{O}-\mathrm{C}_{1}$ diagram of $\mathrm{XY}$ UMa is shown in Fig. 3 . This diagram contains other times of minima which can 


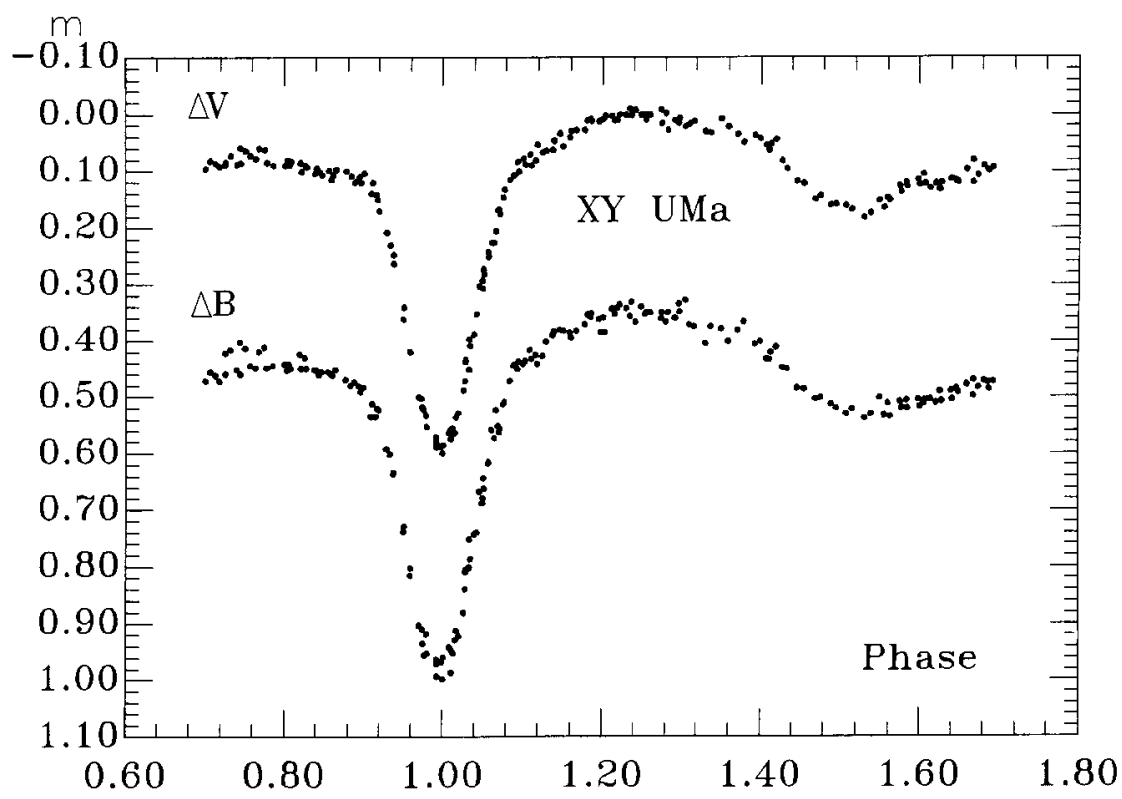

Fig. 1. Differential $B$ and $V$ light curves of XY UMa for 1994

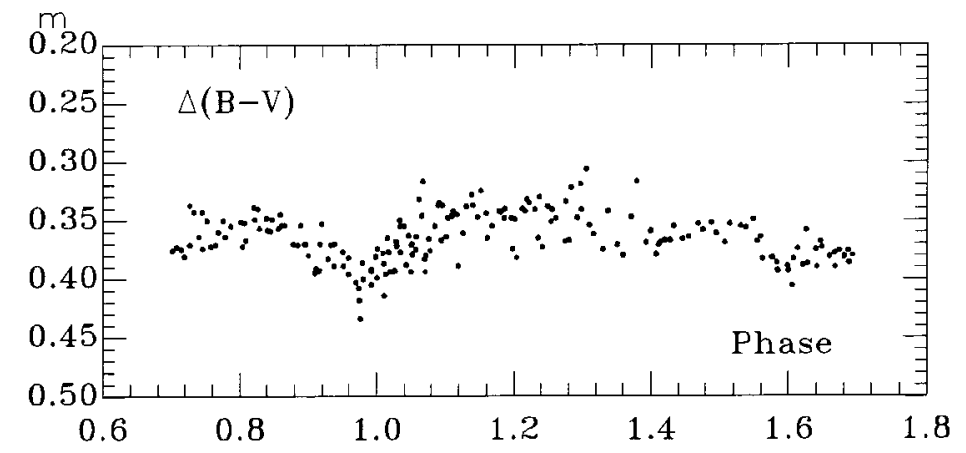

Fig. 2. $\Delta(B-V)$ colour curve of XY UMa for 1994

be found from the literature: Apart from our minima we used all visiual, photographic, and photoelectric minima from the list compiled by Pojmanski \& Geyer (1990), and BBSAG observers, and Hanzl (1991), and two photoelectric minima observed recently by Jeffries et al. (1995). O- $\mathrm{C}_{1}$ deviations in Fig. 3 were calculated with the ephemeris given in formula (1). The arbitrary weights of the points were chosen from Pojmanski \& Geyer (1990). So, small points on this diagram represent uncertain data of weight 0 . Photographic and photoelectric data are represented by filled dots of sizes proportional to their weights. Also, asterisks on this diagram represented the $\mathrm{O}-\mathrm{C}_{1}$ residuals of secondary minima. As it is clearly seen from the figure, the orbital period of XY UMa changes with time. However, as it was stated by Pojmanski \& Geyer (1990), the asymmetry seen in the vicinity of the secondary minimum affects the precise determination of the times of secondary minima, and accordingly, in the period analysis of $\mathrm{XY}$ UMa only the $\mathrm{O}-\mathrm{C}$ residuals per- taining to the times of primary minima were used as it was the case with earlier works.

In order to calculate the phases of the observations of XY UMa, the light elements of the system have been derived by using the photoelectric primary minima times with $E>17000$ cycles (see Fig. 3) as;

$$
\begin{aligned}
& \text { Hel Min I JD }=35216.5298( \pm 0.0017) \\
& \quad+0.47899373\left( \pm 0.710^{-7}\right) \cdot E
\end{aligned}
$$

with the weighted least squares method. In the light curves which were formed using these phases, primary minimum coincides with the phase 0.0 (see Fig. 1).

The $\mathrm{O}-\mathrm{C}$ diagram of XY UMa in Fig. 3 indicates that the orbital period of the system is changing in a sinusoidal character. There are two mechanisms which explain such periodic orbital period variations of eclipsing binary systems: (i) a third body, and (ii) apsidal motion. Apsidal motion is unlikely in the case of XY UMa, since the orbital eccentricity of XY UMa is too small, and the $\mathrm{O}-\mathrm{C}$ 


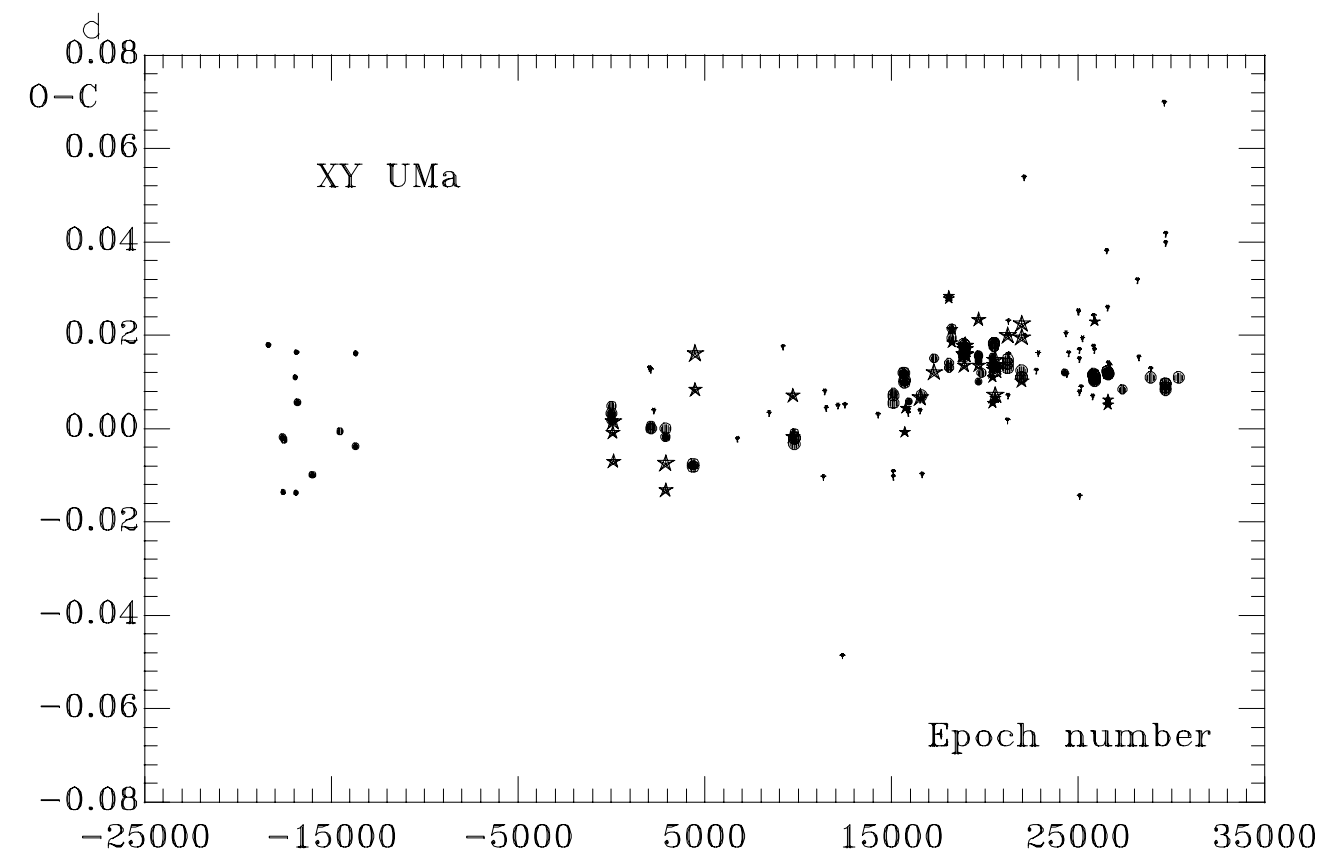

Fig. 3. The $\mathrm{O}-\mathrm{C}$ versus epoch numbers diagram for XY UMa

residuals of primary and secondary minima times do not give a sinusoidal $\mathrm{O}-\mathrm{C}$ curve caused by apsidal motion (i.e, times of secondary minimum of XY UMa in Fig. 3 do not trace out a sinusoidal $\mathrm{O}-\mathrm{C}$ curve of equal amplitude but exactly $180^{\circ}$ out of phase to a sinusoidal O-C curve traced out by times of primary minimum). Therefore, the third body hypothesis can explain the sinusoidal variation of the orbital period of XY UMa. However, if this sinusoidal variation character is, in fact, shape of the orbital period modulation, the Applegate mechanism can explain orbital period modulation of XY UMa. We shall investigate these suggested two models in turn.

\subsection{A third body?}

Such an O-C curve in Fig. 3 could easily be represented with a light-time effect which results from orbiting around a third body. According to third body hypothesis, time delay which are resulting from the movement on the eccentric three body orbit may be calculated as follows (Irwin 1959):

$\Delta t=\frac{a \sin i}{2.59010^{10}}\left\{\frac{1-e^{2}}{1+e \cos v} \sin (v+\omega) e \sin \omega\right\}$

where $a \sin i$ is the projected distance of the third-body to the eclipsing pair (in $\mathrm{km}$ ), $c$ is the speed of light (in $\mathrm{km} /$ day). $e$ is the eccentricity; $v$ is true anomaly; $\omega$ is the longitude of periastron passage of the orbit of three-body system, while $T_{\mathrm{s}}$ is the epoch of periastron passage and $P_{\mathrm{S}}$ is the period of the orbit of three-body system.

The ephemeris for the minimum is now given by:

$\operatorname{Min} \mathrm{I}=T_{0}+P \cdot E+\Delta t$.
Unfortunately, the theoretical $\mathrm{O}-\mathrm{C}$ curve given this formula do not fit to the old photographic data of Geyer (1977). Therefore, a quadratic term $\left(=Q \cdot E^{2}\right)$ was added to the Eq. (4):

$\operatorname{Min} \mathrm{I}=T_{0}+P \cdot E+Q \cdot E^{2}+\Delta t$

where $Q$ is the coefficient of the quadratic term and gives change (increase or decrease) of the orbital period of the eclipsing binary (in day). Finally, using Eq. (5) to observed minima times of XY UMa, we have performed the weighted regression method with differential correction for $T_{0}, P, Q, a \sin i, e, \omega, T_{\mathrm{s}}$, and $P_{\mathrm{s}}$ parameters which are given in Table 3 .

Table 3. Parameters of the third-body orbit

\begin{tabular}{lll}
\hline Parameter & Value & Standart deviation \\
\hline$T_{0}$ HJD & 35216.5039 & 0.0013 \\
$P$ (day) & 0.47899433 & $1.3010^{-7}$ \\
$Q$ (day) & $1.8510^{-11}$ & $3.3810^{-14}$ \\
$a_{12} \sin i(\mathrm{~km})$ & $2.22410^{8}$ & $1.5910^{7}$ \\
$e$ & 0.41 & 0.09 \\
$\omega$ & $53^{\circ}$ & $12^{\circ}$ \\
$T_{\mathrm{s}}$ (cycles) & 16205 & 3739 \\
$P_{\mathrm{s}}$ (cycles) & 24804 & 1168 \\
\hline
\end{tabular}

The $Q$ parameter in Table 3 shows that the orbital period of XY UMa is secularly increasing. The period 


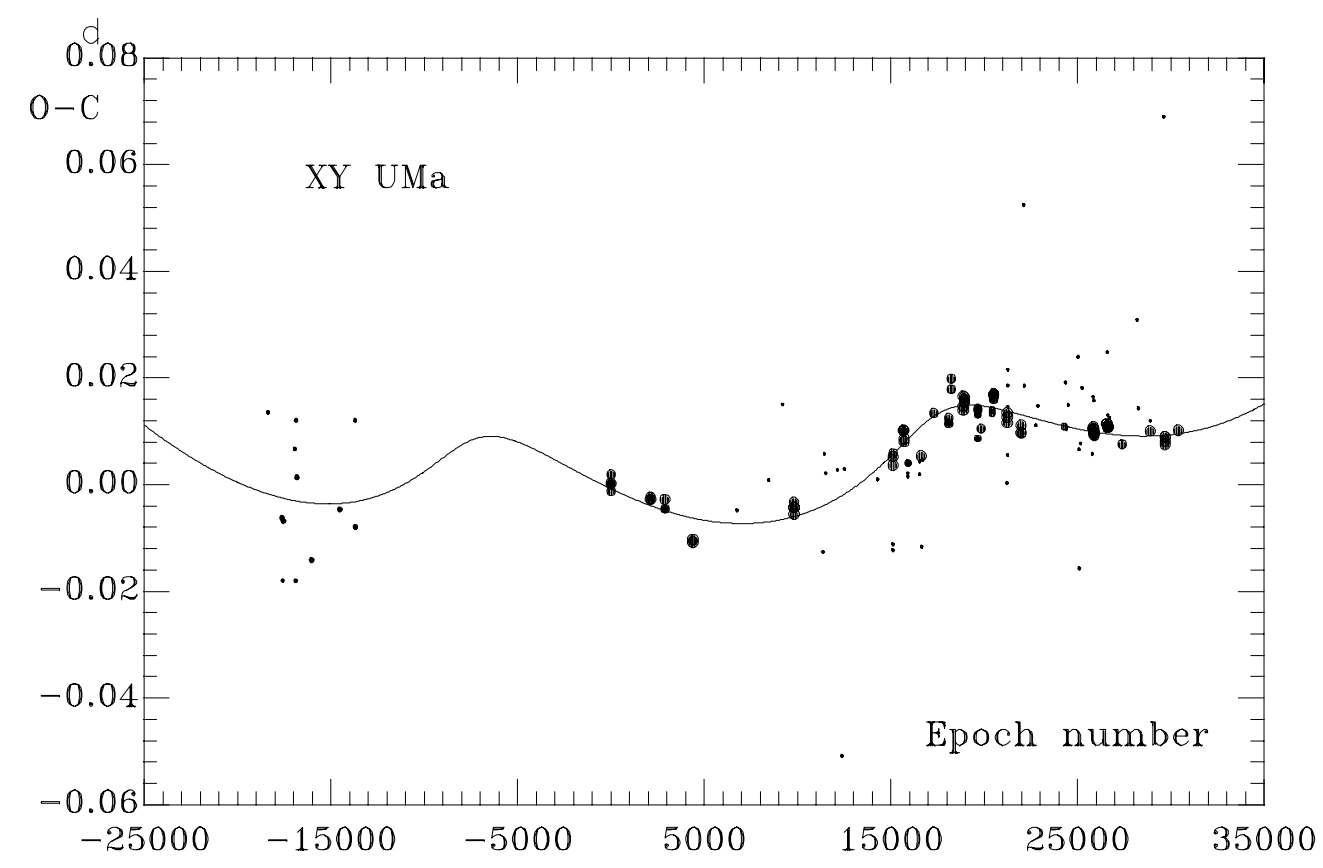

Fig. 4. The $\mathrm{O}-\mathrm{C}$ versus epoch numbers diagram for XY UMa. The residuals were calculated according to linear part of the ephemeris given in Table 3. The continuous line represents an appropriate fit to the data

increase is found to be about $0.24 \pm 0.04 \mathrm{~s}$ per century. The mass transfer and mass loss phenomena can be held responsible for the secular increase seen in the orbital period of the system. Furthermore, existence of MgII emission lines which is believed to be originated from the circumbinary environment (Gurzadyan \& Cholakyan 1995) supports this proposal. The primary component of XY UMa is close to fill out its Roche lobe (see Sect. 4). So, this primary component may be responsible for the mass loss. The orbital period increase of XY UMa and the mass of the mass losing primary component greater than the mass of other component indicates that the only case IV from mass-loss cases discussed by Singh \& Chaubey (1986) is possible to XY UMa. The mass loss in the case IV in which the ejected material escapes out from the binary system may be calculated as follows:

$$
\frac{\dot{P}}{P}=\left\{1-3 \frac{M}{m_{1}}+3 \frac{m_{2}}{m_{1}}\right\} \frac{\dot{m}}{M}
$$

Using Eq. (6) to $m_{1}=0.90 m_{\odot}$ and $m_{2}=0.75 m_{\odot}$ which are given in Sect. 5, we have calculated $4.8610^{-8} m_{\odot} \mathrm{yr}^{-1}$ for the mass loss rate for primary component to observed value $\frac{\dot{P}}{P}=5.8910^{-8} \mathrm{yr}^{-1}$.

The $\mathrm{O}-\mathrm{C}_{3}$ residuals of all observed minima times of XY UMa which were calculated according to the linear part of the ephemeris given in Table 3 are plotted versus epoch numbers in Fig. 4. In this diagram the theoretical $\mathrm{O}-\mathrm{C}$ curve of the light-time effect is also plotted.

The parameters given in Table 3 show that the eclipsing pair revolve around a center of mass of the three body system with a period of $32.5 \mathrm{yr}$. The projected distance of the third-body to the XY UMa is $1.487 \mathrm{AU}$. These values lead to a small mass function of $f\left(m_{3}\right)=0.0031 m_{\odot}$ for the hypothetical third-body. So, the masses of the thirdbody were computed for different values of the inclination of three-body system and are given in Table 4. In this computation $m_{1}=0.90 m_{\odot}$ and $m_{2}=0.75 m_{\odot}$ which are given in Sect. 5 were applied.

Table 4. Mass of the third-body depending on the orbital inclination

\begin{tabular}{ll}
\hline Inclination & $m_{3}\left(m_{\odot}\right)$ \\
\hline $30^{\circ}$ & 0.483 \\
$40^{\circ}$ & 0.361 \\
$50^{\circ}$ & 0.297 \\
$60^{\circ}$ & 0.259 \\
$70^{\circ}$ & 0.237 \\
$80^{\circ}$ & 0.225 \\
$90^{\circ}$ & 0.221 \\
\hline
\end{tabular}

According to Table 4 the minimum mass of the thirdbody was found to be $0.22 m_{\odot}$. This value is different from that found by Pojmanski \& Geyer (1990) for thirdbody hypothesis on XY UMa. The cause of this difference is that Pojmanski and Geyer have been fixed the orbital period on the least square fitting process for the 
light-time effect since the earlier photographic data could not be fit. Instead of this artificial method applied by Pojmanski and Geyer on $\mathrm{O}-\mathrm{C}$ analysis of XY UMa, in this work, a quadratic term was added to Eq. (4) given for light-time effect and all the parameters were left free on fitting process. Such a method could be considered more reliable since it does not include an artificial effect in the process of fitting a theoretical curve to data.

If the relative orbital inclination of three body system is equal to orbital inclination of XY UMa, the semi-major axis $a_{3}$ of the third-body orbit around the center of mass of the triple system is about 11.2 AU. This value shows that the third-body, if it exists, revolves around much beyond the outer Lagrangian points of XY UMa and its orbit should be stable.

If the third-body is a main-sequence star, the massluminosity function $\left(M_{\mathrm{b}}=5.84-6.54 \log M\right)$ for the mainsequence stars with $M<0.7 M_{\odot}$ given by Demircan and Kahraman (1991) gives the bolometric absolute magnitude of the third-body $M_{\mathrm{b}}=10{ }^{\mathrm{m}} 14$. Whereas, the bolometric absolute magnitude of XY UMa is obtained to be about 4.68 in the photometric analysis in this work. So, the third-body is more than about 5.5 fainter than $\mathrm{XY}$ $\mathrm{UMa}$, and then the lines of the third body on the spectrum of XY UMa are unobserved. If one considers the distance to XY UMa as 100 pc given by Dempsey et al. (1993), the separation between the tertiary body and the eclipsing pair may be found as $00^{\prime \prime} 014$. As a result, either the faintness of the third-body in the system or its small separation with the eclipsing pair makes its direct observation with the current equipment almost impossible, leaving the third-body hypothesis in dark.

Zeilik (1997) has found the evidence for a third light from another star in his recent photometric analysis. He stated that if the maculation effect is large, and centered around zero degrees, the timing may be in error. So, he found that one have to use clean light curves to get the correct times for the primary minima in such cases. But, the main problem is, in his work, to correctly determine the effects distorted the light curves, and then to correctly clean the distributive effects from the light curves. It can be clearly seen that the probable errors in the cleaning processes will damage both the naturalness of the light curves and the normal light curves.

\subsection{Orbital period modulation}

At this stage of the orbital period analysis, the cause of the orbital period change in XY UMa is thought to be caused by the period modulation of the sine variation. Therefore, the sinusoid with amplitude $a_{\mathrm{s}}$, period $P_{\mathrm{s}}$ and a moment $T_{\mathrm{s}}$ of minimum was added to the ephemeris $\left(T_{0}\right.$ and $\left.P\right)$ to get the best fit to the data:

$\operatorname{MinI}=T_{0}+P \cdot E+a_{\mathrm{s}} \cdot \sin \left[\frac{2 \pi}{P_{\mathrm{s}}}\left(E-T_{\mathrm{s}}\right)\right]$.
However, a quadratic term $\left(=Q \cdot E^{2}\right)$ was added to this equation since the earlier photographic data could not be fit. So, using the sine equation with a quadratic term to all observed times of minima of XY UMa, we have performed the weighted regression method with differential correction for $T_{0}, P, Q, a_{\mathrm{s}}, T_{\mathrm{s}}$ and $P_{\mathrm{s}}$ parameters. The parameters found are given in Table 5 .

Table 5. Parameters for the XY UMa sinusoidal solution

\begin{tabular}{lll}
\hline Parameter & Value & Standart deviation \\
\hline$T_{0}$ HJD & 35216.5021 & 0.0011 \\
$P$ (days) & 0.47899433 & $0.1310^{-8}$ \\
$Q$ (days) & $2.1110^{-11}$ & $0.3710^{-11}$ \\
$a_{\mathrm{s}}$ (days) & 0.0079 & $4.010^{-4}$ \\
$T_{\mathrm{s}}$ (cycles) & 12953 & 190 \\
$P_{\mathrm{s}}$ (cycles) & 23713 & 615 \\
\hline
\end{tabular}

According to this $Q$ parameter, the increase in the orbital period was found to be $0.28 \pm 0.05 \mathrm{~s}$ per century. This increase in the orbital period is related to the case IV from mass loss cases discussed by Singh \& Chaubey (1986). Accordingly, the mass loss rate for the primary component is estimated to be $5.5410^{-8} m_{\odot} \mathrm{yr}^{-1}$.

The $\mathrm{O}-\mathrm{C}_{4}$ residuals of all observed minima times of XY UMa which were calculated according to the linear part of the ephemeris given in Table 5 are plotted versus epoch numbers in Fig. 5 together with the theoretical $\mathrm{O}-\mathrm{C}$ curve of the sine equation with a quadratic term.

The primary component of XY UMa is a RS CVn type star with a surface magnetic activity. The star-spot activity is evident from the light curve analysis in this work (see Sect. 4). So, for the cause of the orbital period changes seen in the system, the activity in the primary component might be taken responsible and the Applegate gravitational coupling mechanism (Applegate 1992) can explain the sinusoidal variation seen in Fig. 5 .

The Applegate mechanism requires that the active star be variable, and that the period of the luminosity variation be the same as the period of the orbital period modulation. Applegate (1992) explains that maximum luminosity should coincide with an $\mathrm{O}-\mathrm{C}$ curve minimum if the star's outside spins faster than its inside but should coincide with an $\mathrm{O}-\mathrm{C}$ curve maximum if the outside spins slower than the inside. In the case of XY UMa, Geyer (1976) has presented that the average brightness of the binary system changed between 1955 and 1975 in a sinusoidal manner by $0.18 \mathrm{mag}$ in $V$ and $0.20 \mathrm{mag}$ in $B$, indicating a periodic variation of about 28 to 30 years, and that the lowest system brightness was observed in 1961, and the highest in 1975. According to Geyer's paper, the mean brightness and the orbital period of XY UMa vary with 


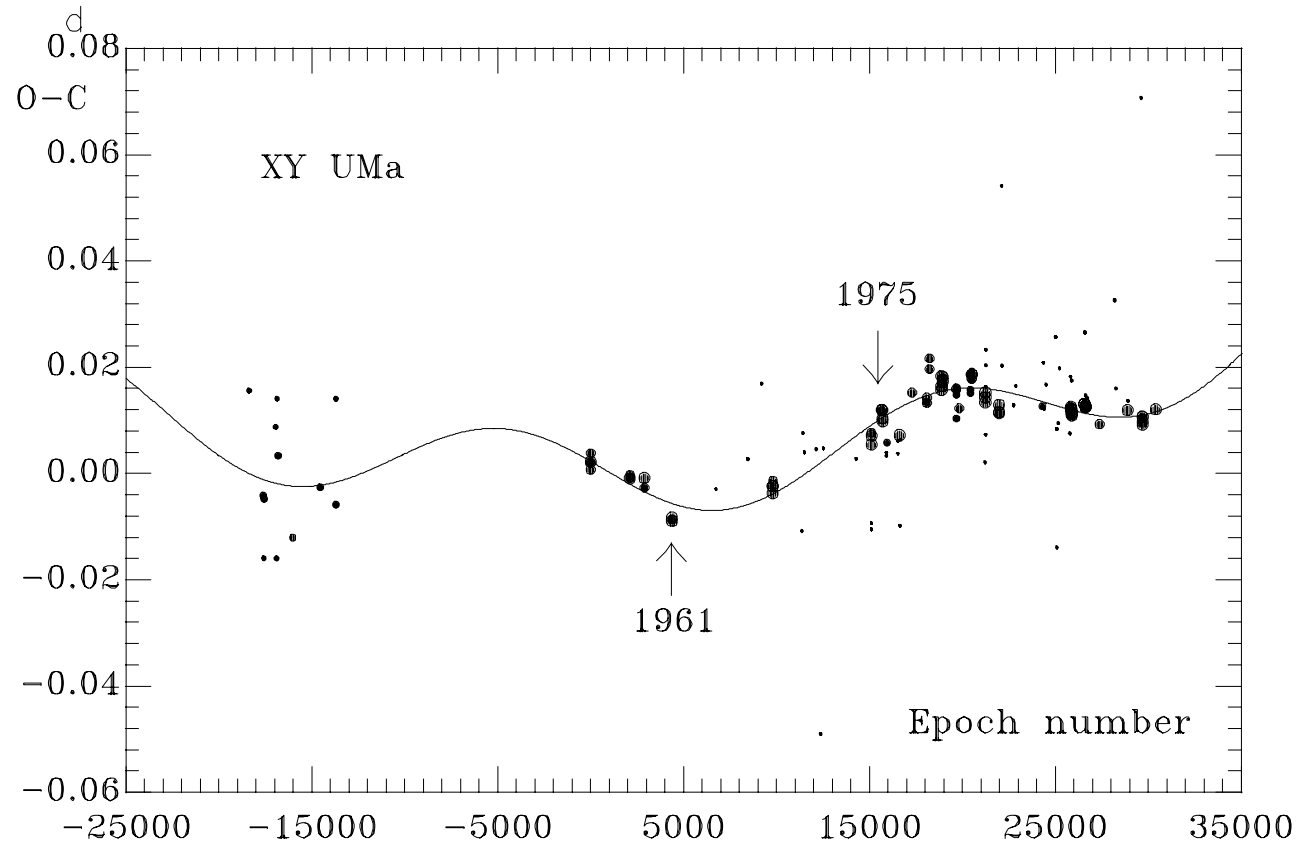

Fig. 5. The $\mathrm{O}-\mathrm{C}$ versus epoch numbers diagram for $\mathrm{XY}$ UMa. The residuals were calculated according to linear part of the ephemeris given in Table 5. The continuous line represents an appropriate fit to the data

the same cycle length, $\sim 30$ years. But, the maximum system brightness in 1975 or minimum system brightness in 1961 does not coincide with the minimum or maximum of $\mathrm{O}-\mathrm{C}$ curve in Fig. 5. However, due to the big asymmetries in the light curves of the system and insufficient data, this conclusion could not be taken as final. Only systematic and continuous observations may help to decide on the existence of any connection between the mean brightness and period variations.

Here, we use Applegate's formalization in an effort to examine the orbital period changes of XY UMa. We assume that the characteristics of the active G2-G5V component of the system are: $m=0.90 m_{\odot}, R=1.08 R_{\odot}$, and $\frac{a}{R}=2.816$ given in Sect. 5 .

(i) The $\mathrm{O}-\mathrm{C}_{4}$ diagram of $\mathrm{XY}$ UMa in Fig. 5 shows a modulation with a semi-amplitude of $\mathrm{O}-\mathrm{C}=0.0079$ day and a modulation period of $P_{\bmod }=31.1 \mathrm{yrs}$. This gives the observed orbital period change of $\Delta P=$ $0.181 \mathrm{~s}$. (ii) The angular momentum transfer, which produces the 0.181 second period change, has been computed to be $2.1910^{47} \mathrm{~g} \mathrm{~cm}^{2} \mathrm{~s}^{-1}$. (iii) The moment of inertia of the shell has been computed to be $6.78010^{53} \mathrm{gcm}^{2}$ by assuming the mass of the shell is one-tenth of the active component's mass. (iv) The variable part of the differential rotation is $2.12510^{-3}$. (v) The energy required to transfer the angular momentum is $1.41510^{41} \mathrm{ergs}$. (vi) If the energy requirement is supplied by the nuclear luminosity of the star with no energy storage in the convection zone, the star will be variable with the RMS luminosity variation of $\Delta L=0.117 L_{\odot}$ which give rise to only about $\sim 0.10$ mag light variation of the system. (vii) The mean subsurface magnetic field which is provide the RMS torque required to periodically exchange $\Delta J$ between the outer shell and inner part of the star is obtained to be about $10 \mathrm{k}$ Gauss.

If the hypothesized magnetic cycle can be estimated from an $\mathrm{O}-\mathrm{C}$ diagram as the time between one period decrease and the next (Hall 1990), the $\mathrm{O}-\mathrm{C}_{4}$ diagram for $\mathrm{XY}$ UMa in Fig. 5 gives the magnetic cycle of the primary component as 31.1 yrs. This value agrees with the magnetic cycle for solar-type dwarfs computed by Hall (1990). Finally, the Applegate model gives reasonable solution to the orbital perid modulation of XY UMa which is caused by the magnetic activity of the primary component.

\section{Photometric analysis}

The strong curve asymmetry appeared as the brightness difference between the light maxima in the light curves of XY UMa in Fig. 1. Such asymmetric light curves of the system observed by Geyer (1976) and are interpreted as stellar star-spot activity of the primary component of this binary. Geyer proposed the following model for the interpretation of this phenomena. The spotted area of the G2V component of XY UMa was on the far side of the observer during primary minimum. Thus a larger light loss was caused by the transit eclipse of the smaller component. Under these conditions the observer, outside of the primary eclipse, always sees parts of the spotted area, and this phenomenon can be well observed shortly after the secondary component is occulted. This causes the plateau shaped part of the light curve, and at the same time the 
system brightness is at its lowest value. In this work, we took into consideration this model proposed by Geyer.

The synthetic light curve technique of Wilson \& Devinney (1971) was applied to the system XY UMa. This method has been described by many authors. The modern version of the method developed by Wilson (private communication, 1992) was used for our solutions. The major revision is that of 1992, which has detalied reflection and non-linear limb darkening (both optional), adjustment of spot parameters, an optional provision for spots to move with the rotating surface, capability for following light curve development over large numbers of orbits, and greater speed. The method assumes the star surfaces to be equipotentials and computes the light curve as a function of the following parameters: the orbital inclination $i$, surface potentials $\Omega_{\mathrm{h}, \mathrm{c}}$, flux-weighted average surface temperatures $T_{\mathrm{h}, \mathrm{c}}$, mass ratio $q=m_{\mathrm{c}} / m_{\mathrm{h}}$, unnormalized monochromatic luminosities $L_{\mathrm{h}, \mathrm{c}}$, limb-darkening coefficients $x_{\mathrm{h}, \mathrm{c}}$, gravity-darkening exponents $g_{\mathrm{h}, \mathrm{c}}$, bolometric albedos $A_{\mathrm{h}, \mathrm{c}}$. Throughout this paper the subscripts $h$ and $c$ refer to the primary (hotter) and secondary (cooler) component, respectively. Also, this model can computing optionally the following star-spot parameters: the latitude of a star-spot center $\lambda$, the longitude of a star-spot center $\beta$, the angular radius of a star-spot $\gamma$, and the temperature factor of a spot $\tau_{\text {spot }}$.

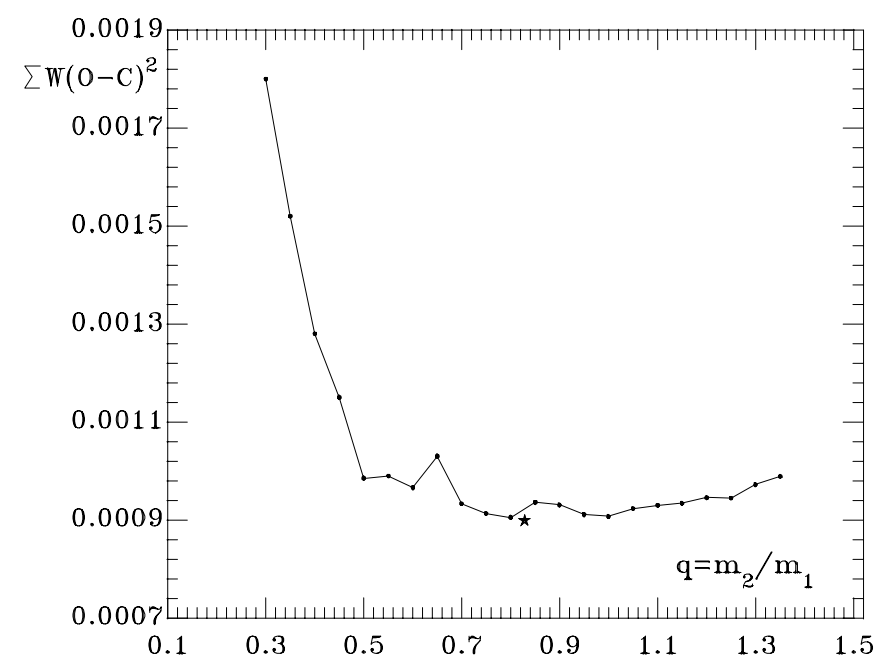

Fig. 6. The behaviour $\Sigma W(\mathrm{O}-\mathrm{C})^{2}$ as a function of mass ratio $q$

For the solution, the 197 observational points were combined into 50 normal points in each colour, and weighted directly according to the number of individual observations included in a point. The normal points were given in Table 6 . The light curves were analyzed independently for $B$ and $V$ colours. The temperature of primary component was taken from Budding \& Zeilik (1987) as equal to $5700 \mathrm{~K}$ corresponding to the G5 spectral class
Table 6. Normal points of XY UMa

\begin{tabular}{llllll}
\hline Phase & $\Delta B$ & $\Delta V$ & $l_{B}$ & $l_{V}$ & $W^{*}$ \\
\hline 0.7065 & 0.4710 & 0.0950 & 0.8971 & 0.9186 & 1 \\
0.7359 & 0.4411 & 0.0795 & 0.9220 & 0.9317 & 10 \\
0.7813 & 0.4364 & 0.0759 & 0.9260 & 0.9348 & 7 \\
0.8316 & 0.4466 & 0.0926 & 0.9175 & 0.9206 & 11 \\
0.8789 & 0.4659 & 0.1065 & 0.9013 & 0.9090 & 8 \\
0.9039 & 0.4865 & 0.1115 & 0.8845 & 0.9048 & 2 \\
0.9155 & 0.5225 & 0.1295 & 0.8558 & 0.8900 & 2 \\
0.9230 & 0.5250 & 0.1537 & 0.8538 & 0.8705 & 3 \\
0.9343 & 0.5910 & 0.2080 & 0.8037 & 0.8282 & 1 \\
0.9419 & 0.6233 & 0.2467 & 0.7802 & 0.7994 & 3 \\
0.9560 & 0.7380 & 0.3610 & 0.7024 & 0.7198 & 1 \\
0.9566 & 0.7290 & 0.3400 & 0.7082 & 0.7338 & 1 \\
0.9646 & 0.8085 & 0.4195 & 0.6584 & 0.6822 & 2 \\
0.9753 & 0.9030 & 0.5000 & 0.6038 & 0.6337 & 1 \\
0.9823 & 0.9342 & 0.5250 & 0.5868 & 0.6194 & 5 \\
0.9972 & 0.9710 & 0.5790 & 0.5673 & 0.5894 & 1 \\
0.9975 & 0.9785 & 0.5795 & 0.5634 & 0.5892 & 2 \\
0.0052 & 0.9750 & 0.5903 & 0.5652 & 0.5834 & 3 \\
0.0159 & 0.9560 & 0.5623 & 0.5751 & 0.5986 & 4 \\
0.0228 & 0.9207 & 0.5420 & 0.5941 & 0.6098 & 3 \\
0.0334 & 0.8335 & 0.4575 & 0.6435 & 0.6589 & 4 \\
0.0407 & 0.7705 & 0.4113 & 0.6817 & 0.6874 & 4 \\
0.0496 & 0.7035 & 0.3275 & 0.7249 & 0.7423 & 2 \\
0.0581 & 0.6502 & 0.2747 & 0.7612 & 0.7791 & 6 \\
0.0685 & 0.5650 & 0.2260 & 0.8231 & 0.8147 & 2 \\
0.0748 & 0.5475 & 0.1793 & 0.8364 & 0.8503 & 4 \\
0.0820 & 0.5100 & 0.1390 & 0.8656 & 0.8823 & 2 \\
0.0976 & 0.4464 & 0.0974 & 0.9175 & 0.9165 & 5 \\
0.1246 & 0.4192 & 0.0692 & 0.9407 & 0.9406 & 10 \\
0.1764 & 0.3714 & 0.0253 & 0.9829 & 0.9792 & 10 \\
0.2244 & 0.3538 & 0.0024 & 0.9988 & 1.0000 & 11 \\
0.2743 & 0.3526 & 0.0057 & 1.0000 & 0.9970 & 9 \\
0.3187 & 0.3619 & 0.0190 & 0.9915 & 0.9849 & 7 \\
0.3757 & 0.3864 & 0.0302 & 0.9695 & 0.9748 & 5 \\
0.4129 & 0.4223 & 0.0528 & 0.9381 & 0.9549 & 4 \\
0.4321 & 0.4363 & 0.0733 & 0.9261 & 0.9370 & 3 \\
0.4511 & 0.4840 & 0.1180 & 0.8865 & 0.8994 & 1 \\
0.4673 & 0.4945 & 0.1360 & 0.8780 & 0.8847 & 2 \\
0.4808 & 0.5010 & 0.1430 & 0.8728 & 0.8790 & 1 \\
0.4969 & 0.5155 & 0.1590 & 0.8613 & 0.8663 & 2 \\
0.5166 & 0.5255 & 0.1645 & 0.8534 & 0.8619 & 2 \\
0.5397 & 0.5335 & 0.1780 & 0.8472 & 0.8513 & 2 \\
0.5572 & 0.5165 & 0.1580 & 0.8605 & 0.8670 & 2 \\
0.5659 & 0.5225 & 0.1490 & 0.8558 & 0.8742 & 2 \\
& 0.5133 & 0.1275 & 0.8630 & 0.8916 & 4 \\
& 0.5040 & 0.1150 & 0.8704 & 0.9019 & 1 \\
0.6790 & 0.4925 & 0.1160 & 0.8796 & 0.9011 & 4 \\
0.4730 & 0.0930 & 0.8955 & 0.9203 & 1 \\
\hline & & & & &
\end{tabular}

*: weights directly according to the number of individual observations included in a point. 
Table 7. Geometrical and physical elements of the star-spot of primary component of XY UMa and the other photometric parameters obtained from solution of $V$ light curve

\begin{tabular}{|c|c|c|c|c|c|c|}
\hline $\begin{array}{l}\text { latitude of } \\
\text { spot center } \\
\lambda\end{array}$ & $\begin{array}{l}\text { longitude of } \\
\text { spot center } \\
\beta\end{array}$ & $\begin{array}{l}\text { angular radius of } \\
\text { the spot } \\
\gamma\end{array}$ & $\begin{array}{l}\text { temperature factor } \\
\text { of the spot } \\
\tau_{\text {spot }}\end{array}$ & PSHIFT & $i$ & $T_{\mathrm{c}}$ \\
\hline $\begin{array}{l}90^{\circ} \\
\pm 5^{\circ}\end{array}$ & $\begin{array}{l}104^{\circ} \\
\pm 1^{\circ}\end{array}$ & $\begin{array}{l}16^{\circ} .2 \\
\pm 0.11\end{array}$ & $\begin{array}{l}0.670 \\
\pm 0.015\end{array}$ & $\begin{array}{l}0.0028 \\
\pm 0.0003\end{array}$ & $\begin{array}{l}76.3 \\
\pm 0.2\end{array}$ & $\begin{array}{l}3871 \mathrm{~K} \\
\pm 47\end{array}$ \\
\hline$\overline{\Omega_{\mathrm{h}}}$ & $\Omega_{\mathrm{c}}$ & $q$ & $L_{\mathrm{h}} /\left(L_{\mathrm{h}}+L_{\mathrm{c}}\right)$ & $L_{\mathrm{c}} /\left(L_{\mathrm{h}}+L_{\mathrm{c}}\right)$ & $\Sigma W(\mathrm{O}-\mathrm{C})^{2}$ & \\
\hline $\begin{array}{l}3.5981 \\
\pm 0.0145\end{array}$ & $\begin{array}{l}4.0073 \\
\pm 0.0274\end{array}$ & $\begin{array}{l}0.75 \\
\text { (fixed) }\end{array}$ & $\begin{array}{l}0.9430 \\
\pm 0.0057\end{array}$ & 0.0570 & 0.0009 & \\
\hline
\end{tabular}

given in literature. The limb-darkening coefficients were taken from Jassur (1986), the gravity-darkening exponents from Lucy (1967). The bolometric albedos $A_{\mathrm{h}}$ and $A_{\mathrm{c}}$ were set to be equal to 0.5 from Rucinski (1969) for convective atmospheres. These parameters were kept constant during all the iterations.

As a first step in the photometric analysis of the system, the value of the $q$ parameter was taken to be 0.75 which is very close to the one given by Geyer (1977). The purpose here is to determine the parameters of the spot with the Wilson-Devinney code, which is supposed to take place on the G2-G5V component causing an asymmetry in the light curves of the system XY UMa (see Fig. 1). Wilson-Devinney code needs spot parameters predicted closely. Otherwise, to converge to a meaningful solution is very hard to achieve. Therefore, we have calculated unspotted light curve for $V$ colour of XY UMa by using geometrical and physical parameters of the system from Geyer (1977) and Jassur (1986). This unspotted $V$ light curve has been subtracted from observed $V$ light curve. This difference provided as a rough light curve which has only the effects of the spots. The spot parameters are then predicted roughly by fitting a synthetic light curve to this difference curve. The synthetic light curve which is used to predict rough spot parameters is computed according to Eker (1994). This rough spot parameters were then adopted for Wilson-Devinney code. This is analyzed for maculation (star-spot) effects. Only one spot group was used. So, the search was made in the observed $V$ light curve by choosing $\lambda, \beta, \gamma, \tau_{\mathrm{spot}}, \mathrm{i}, T_{\mathrm{c}}, \Omega_{\mathrm{h}}, \Omega_{\mathrm{c}}$, and $L_{\mathrm{h}}$ as adjustable parameters in Wilson-Devinney program. The analysis was made with detached configuration (i.e. Mode 2 ). The parameters thus obtained were given in Table 7 . The probable error of each adjustable parameter given in Table 7 was calculated while all the other parameters were fixed to values given in the table.

Using the Wilson-Devinney (1992 version) program, we found that a circular dark spot which covers $\sim 7 \%$ of stellar photosphere of primary component (see Table 7)
Table 8. Photometric solutions of XY UMa

\begin{tabular}{|c|c|c|}
\hline Parameters & Blue $(B)$ & Yellow $(V)$ \\
\hline $\begin{array}{l}\text { Latitude of spot center } \lambda \\
\text { Longitude of spot center } \beta \\
\text { Angular radius of the spot } \gamma \\
\text { Temperature factor of the spot } \tau_{\text {spot }}\end{array}$ & $\begin{array}{l}89.890 \\
103.460 \\
16.190 \\
0.609\end{array}$ & $\begin{array}{l}89.890 \\
103.460 \\
16.190 \\
0.670\end{array}$ \\
\hline $\begin{array}{l}a \\
e \\
\omega\end{array}$ & $\begin{array}{l}3.041 R_{\odot} \\
0.00 \\
90^{\circ}\end{array}$ & $\begin{array}{l}3.041 R_{\odot} \\
0.00 \\
90^{\circ}\end{array}$ \\
\hline$i$ & $\begin{array}{l}76.107 \\
\pm 0.307\end{array}$ & $\begin{array}{l}75^{\circ} .660 \\
\pm 0.358\end{array}$ \\
\hline $\begin{array}{l}x_{\mathrm{h}} \\
x_{\mathrm{C}} \\
A_{\mathrm{h}} \\
A_{\mathrm{c}} \\
g_{\mathrm{h}} \\
g_{\mathrm{c}}\end{array}$ & $\begin{array}{l}0.750 \\
0.900 \\
0.500 \\
0.500 \\
0.320 \\
0.320\end{array}$ & $\begin{array}{l}0.600 \\
0.800 \\
0.500 \\
0.500 \\
0.320 \\
0.320\end{array}$ \\
\hline$T_{\mathrm{h}}$ & $5700 \mathrm{~K}$ & $5700 \mathrm{~K}$ \\
\hline$T_{\mathrm{C}}$ & $\begin{array}{l}3873 \mathrm{~K} \\
\pm 73\end{array}$ & $\begin{array}{l}3845 \mathrm{~K} \\
\pm 50\end{array}$ \\
\hline$\Omega_{1}$ & $\begin{array}{l}3.6897 \\
\pm 0.0140\end{array}$ & $\begin{array}{l}3.7287 \\
\pm 0.0362\end{array}$ \\
\hline$\Omega_{2}$ & $\begin{array}{l}4.2279 \\
\pm 0.0426\end{array}$ & $\begin{array}{l}4.2047 \\
\pm 0.0402\end{array}$ \\
\hline$q$ & 0.828 & $\begin{array}{l}0.828 \\
\pm 0.014\end{array}$ \\
\hline$L_{\mathrm{h}} /\left(L_{\mathrm{h}}+L_{\mathrm{c}}\right)$ & $\begin{array}{l}0.9663 \\
\pm 0.0066\end{array}$ & $\begin{array}{l}0.9413 \\
\pm 0.0076\end{array}$ \\
\hline$L_{\mathrm{c}} /\left(L_{\mathrm{h}}+L_{\mathrm{c}}\right)$ & 0.0337 & 0.0587 \\
\hline $\begin{array}{l}r_{\mathrm{h}}(\text { pole }) \\
r_{\mathrm{h}} \text { (point) } \\
r_{\mathrm{h}} \text { (side) } \\
r_{\mathrm{h}} \text { (back) }\end{array}$ & $\begin{array}{l}0.3440 \\
0.4013 \\
0.3580 \\
0.3780\end{array}$ & $\begin{array}{l}0.3396 \\
0.3919 \\
0.3528 \\
0.3715\end{array}$ \\
\hline $\begin{array}{l}r_{\mathrm{c}}(\text { pole }) \\
r_{\mathrm{c}} \text { (point) } \\
r_{\mathrm{c}} \text { (side) } \\
r_{\mathrm{c}} \text { (back) }\end{array}$ & $\begin{array}{l}0.2608 \\
0.2795 \\
0.2661 \\
0.2747\end{array}$ & $\begin{array}{l}0.2627 \\
0.2820 \\
0.2681 \\
0.2770\end{array}$ \\
\hline$\Sigma W(\mathrm{O}-\mathrm{C})^{2}$ & 0.0015 & 0.0009 \\
\hline
\end{tabular}




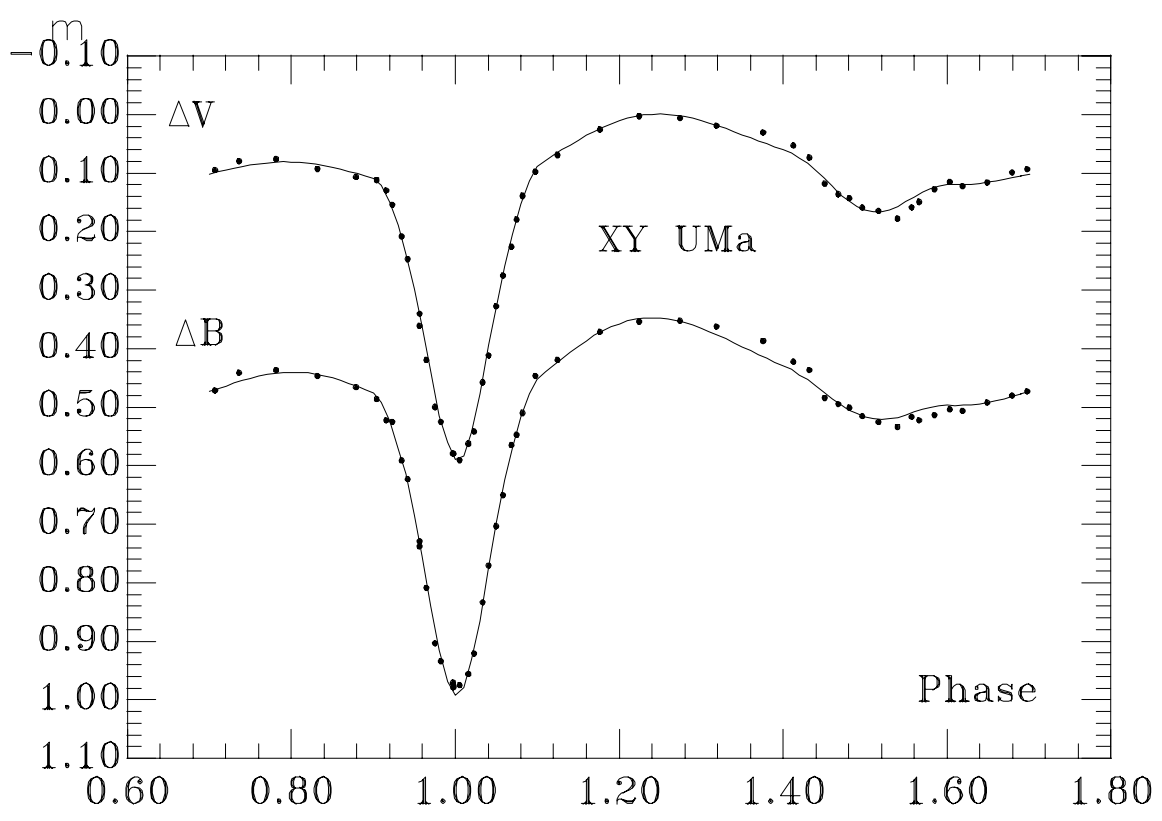

Fig. 7. Normal points of XY UMa in magnitudes, and theoretical light curves corresponding to the parameters obtained from the solutions $B$ and $V$ light curves. Dots and solid lines denote normal points and theoretical light curves, respectively

could account for the intrinsic light variations of the system. The temperature factor of the spot, $\tau_{\text {spot }}$, computed by this program shows that the spot temperature equals to about $3815 \mathrm{~K}$ for a primary star temperature of $5700 \mathrm{~K}$.

At later steps, the spot parameters given in Table 7 were considered to be fixed in order to make things easier and then the light curves were freed off the disturbing effects of the spots.

There is no spectroscopic mass ratio for XY UMa in the literature and in order to test the photometric mass ratio given in the literature, a photometric q-search was performed. The search was made in the $V$ light curve by choosing $i, T_{\mathrm{c}}, \Omega_{\mathrm{h}}, \Omega_{\mathrm{c}}$, and $L_{\mathrm{h}}$ as adjustable parameters. The analysis was made with detached configuration (i.e. MODE 2). The weighted sum of the squared residuals, $\Sigma W(\mathrm{O}-\mathrm{C})^{2}$, for the corresponding mass ratios are shown in Fig. 6. As can be seen from the figure, the variation of the weighted sum of the squared residuals versus mass ratio gives a minimum around $q=0.80$. Therefore, we used this value of $q$ as a starting input parameter in the final solution. The convergent solutions were obtained with the free parameters by iterating, as usual, until the corrections on the parameters became smaller than the corresponding probable errors. The mass ratio was found as $0.83 \pm 0.01$ from solution of $V$ light curve. But in the successive iterations in the $B$ light curve, the value of $q$ increased continuously and a meaningful solution could not be obtained. Therefore, the value of $\mathrm{q}$ obtained from the solution of $V$ light curve was adopted and kept fixed in the solution of $B$ light curve, then the other parameters were calculated. The results of final solutions are given in Table 8 . The theoretical light curves calculated with the final el- ements given in Table 8 are shown in Fig. 7. As it seen, they agree quite well. But, the theoretical $B$ light curve slightly differs from observations around the mid-primary.

The configuration of the XY UMa calculated with the Roche model is shown in Fig. 8. Accordingly, the system $\mathrm{XY} \mathrm{UMa}$ is a detached binary.

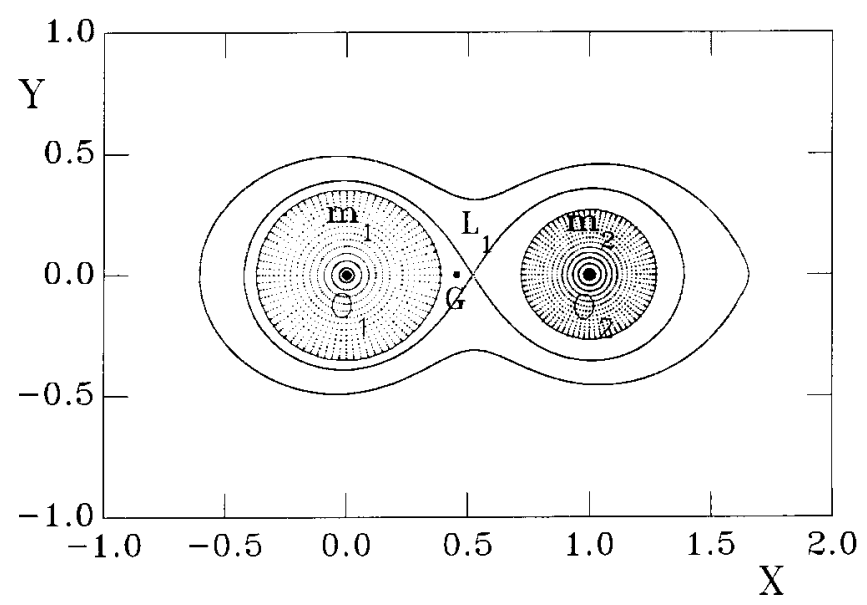

Fig. 8. The configuration of XY UMa for $q=0.828$

\section{Conclusion}

Our study shows that the orbital period variations of XY UMa can be explained in principle by the appealing combination of the Applegate gravitational coupling mechanism and the mass-loss assumption. Existence of MgII emission 
Table 9. Absolute dimensions of XY UMa

\begin{tabular}{lll}
\hline Parameters & $\begin{array}{l}\text { Primary } \\
\text { component }\end{array}$ & $\begin{array}{l}\text { Secondary } \\
\text { component }\end{array}$ \\
\hline Mass $\left(m / m_{\odot}\right)$ & 0.90 & 0.75 \\
Mean Radius $\left(R / R_{\odot}\right)$ & 1.08 & 0.82 \\
$M_{\text {bol }}$ & $4^{\mathrm{m}} 68$ & $6^{\mathrm{m}} 99$ \\
$\log g(\mathbf{c g s})$ & 4.33 & 4.48 \\
\hline
\end{tabular}

lines which is believed to be originated from the circumbinary environment (Gurzadyan \& Cholakyan 1995) supports this mass-loss assumption. In order to verify the Applegate mechanism, however, the magnetic activity of the primary component of the system XY UMa should be monitored together with the $(\mathrm{O}-\mathrm{C})$ variation and luminosity variation of the system with the same time scale.

The asymmetric light curves of XY UMa are obtained in this work. Using the Wilson-Devinney program (Wilson 1992), we determined a circular dark spot which is supposed to take place on the G2-G5V component of the system causing an asymmetry in the light curves of the system. The photometric analysis shows that XY UMa is a detached binary where the primary component is an evolved object and occupies $92 \%$ of its Roche lobe. These results agree with the conclusion of Geyer (1977). Further, the value of the semi-major axis of the apparent orbit of the system (equals to $3.041 R_{\odot}$; Geyer 1977 ) was used to calculate the absolute parameters of the components and the results are given in Table 9 .

Acknowledgements. The authors express their sincere thanks to Ömer Lütfi Deg̃irmenci, Ahmet Devlen, Cengiz Sezer, Ömür Gülmen, Zeki Eker, Varol Keskin and Can Akan for many helpful discussions and valuable suggestions. This work was supported by The Research Foundation of Ege University under 1994/Fen/017.

\section{References}

Applegate J.H., 1992, ApJ 385, 621

Arevalo M.J., Lazaro C., 1990, AJ 99, 983

Baliunas S.L., Vaughan A.H., 1985, ARA\&A 23, 379

Banks T., Budding E., 1989, IAU IBVS No. 3304

Bedford D.K., Jeffries R.D., Geyer E.H., Vilhu O., 1990, MNRAS 243, 557

Bedford D.K., Geyer E.H., 1986, Mitteil. Astron. Gesellschaft, 67,305
Budding E., Kadouri T.H., 1982, Ap\&SS 88, 453

Budding E., Zeilik M., 1987, ApJ 319, 827

Budding E., 1977, Ap\&SS 48, 207

Demircan O., Kahraman G., 1991, Ap\&SS 181, 313

Dempsey R.C., Linsky J.L., Fleming T.A, Schmitt J.H.M.M., 1993, Astrophys. J. Suppl. Ser. 86, 599

Drake S.A., Simon T., Linsky J.L., 1986, AJ 91, 1229

Eker Z., 1994, ApJ 420, 373

Geyer E.H., Hoffmann M., 1981, Mitteil. Astron. Gesellschaft, 52,70

Geyer E.H., Metz K., 1977, Ap\&SS 52, 351

Geyer E.H., Kippenhahn R., Strohmeier W., 1955, Kleine Veroffentl. Remeis Sternwarte Bamberg, No. 9

Geyer E.H., 1976, in Structure and Evolution of Close Binary Systems, by Eggleton P. et al. (ed.) p. 313

Geyer E.H., 1977, Ap\&SS 48, 137

Geyer E.H., 1980, in Close Binary Stars: Observations and Interpretations, Plavec J.M., Papper D.M., Ulrich R.K. (eds.) p. 423

Gurzadyan G.A., 1987, Ap\&SS 123, 67

Gurzadyan G.A., Cholakyan V.G., 1995, Ap\&SS 229, 185

Hall D.S., Kreiner J.M., 1980, Acta Astron. 30, 387

Hall D.S., 1990, in Active Close Binaries, Íbanog̃lu C. (ed.) Dordrecht: Kluwer, p. 95

Hanzl D., 1991, IAU IBVS No. 3615

Heckert P., Zeilik M., 1988, IAU IBVS No. 304.

Hilditch R.W., Bell S.A., 1994, MNRAS 267, 1081

Huisang T., Xuefu L., 1987, A\&A 172, 74

Irwin J.B., 1959, ApJ 64, 149

Jassur D.M.Z., 1986, Ap\&SS 128, 369

Jeffries R.D., Collins C., Elliot K.H., Pittard J.M., Taylor S.B., 1995, IAU IBVS No. 4277

Lee W.B., 1985, Dr. Thesis, Univ. Bonn

Lorenzi L., Scaltriti F., 1977, Acta Astron. 27, 273

Lucy L.B., 1967, Z. Astrophys. 65, 89

Mitrou C.K., Doyle J.G., Mathioudakis M., and Antonopoulou, E., 1996, A\&AS 115, 61

Morris D.H., Mutel R.L., 1988, AJ 95, 204

Pojmanski G., Geyer E.H., 1990, Acta Astron. 40, 245

Qisheng L., Xiaoyu Z., Rhongxian Z., 1989, IAU IBVS No. 3374

Rainger P.P., Hilditch R.W., Edwin R.P., 1991, MNRAS 248, 168

Rucinski S.M., 1969, Acta Astron. 19, 245

Singh M., Chaubey U.S., 1986, Ap\&SS 124, 389

Wilson R.E., Devinney E.J., 1971, ApJ 166, 605

Wilson R.E., 1992 (private communication)

Zeilik M., Batuski D., Elston R., et al., 1982, IAU IBVS No. 2169

Zeilik M., Cox D., De Blasi C., et al., 1988, IAU IBVS No. 3253

Zeilik M., 1997 (private communication) 